\title{
La fiabilité : une voie vers l'équité ?
}

Reliability: A route to equity?

Confianza y responsabilidad: ¿un camino hacia la equidad?

Louis M. Gomez, Anthony S. Bryk et Angel Bohannon

Traducteur : Sylvaine Herold

\section{(2) OpenEdition}

1 Journals

Édition électronique

URL : https://journals.openedition.org/ries/9447

DOI : 10.4000/ries.9447

ISSN : 2261-4265

Éditeur

France Education international

Édition imprimée

Date de publication : 30 avril 2020

Pagination : 195-204

ISBN : 978-2-85420-626-5

ISSN : $1254-4590$

\section{Référence électronique}

Louis M. Gomez, Anthony S. Bryk et Angel Bohannon, «La fiabilité : une voie vers l'équité ? », Revue internationale d'éducation de Sèvres [En ligne], 83 | avril 2020, mis en ligne le 17 juin 2020, consulté le 25 juin 2021. URL : http://journals.openedition.org/ries/9447 ; DOI : https://doi.org/10.4000/ries.9447 


\title{
La fiabilité : une voie vers l'équité ?*
}

\author{
Louis M. Gomez \\ UCLA et Carnegie Foundation \\ for the Advancement of Teaching \\ Anthony S. Bryk \\ Carnegie Foundation \\ for the Advancement of Teaching \\ Angel Bohannon \\ Northwestern University
}

\section{LES INNOVATIONS SE SUCCÈdENT MAIS LES INÉGALITÉS PERSISTENT}

Il est devenu trop habituel de considérer les résultats scolaires décevants des minorités raciales et des personnes économiquement défavorisées comme le résultat malencontreux, mais inévitable, des inégalités structurelles imprégnant la vie sociale, au niveau local, aux États-Unis comme ailleurs. Les structures sociales, telles que la ségrégation dans le logement et la pauvreté intergénérationnelle, ont certainement à voir avec ces résultats. De fait, certains pensent qu'il est pratiquement impossible de parvenir à l'équité en matière éducative, si nos sociétés ne parviennent pas à abattre ni à éradiquer les inégalités structurelles. La croyance en l'impact déterminant des facteurs structurels sur les résultats éducatifs souligne en partie l'inefficacité relative des réformes, des interventions et des services éducatifs offerts. Les services et les innovations en éducation sont importants pour l'équité des résultats scolaires ; nous pensons qu'ils devraient l'être encore davantage à l'avenir. Nous examinerons ici brièvement de quelle façon l'offre de services éducatifs pourrait être conçue et élaborée afin de constituer un levier plus efficace pour parvenir à l'équité.

Car au bout du compte, sur le terrain, l'éducation consiste principalement à créer et à fournir des services. Nous appelons la plupart du temps ces services interventions ou innovations. Un service, tel que nous l'entendons ici, est un système de procédures, d'outils, de politiques et de routines visant à aider les gens à travailler. Par exemple, un curriculum, en tant que service, est un ensemble de contenus précis accompagnés d'outils (par exemple, des évaluations et des activités) et d'un ensemble de spécifications sur la façon dont le curriculum est lié aux normes et procédures locales ou nationales, suggérant comment les enseignants et les élèves peuvent interagir dans le cadre qu'il définit. De même, une technologie éducative innovante est plus qu'une simple technologie et comprend les modalités liées à son utilisation.

* Article traduit par Sylvaine Herold. 
D'une certaine façon, on peut considérer que l'objectif du secteur éducatif est de créer des services favorisant les conditions nécessaires pour que les individus puissent atteindre des résultats à court terme, comme l'apprentissage d'une matière et l'obtention d'un diplôme, et des réalisations à plus long terme, comme la participation à la démocratie, la productivité économique à travers l'emploi et le bonheur, individuellement et de par nos rôles sociaux et culturels. Les disparités systémiques sont la preuve que les services que nous avons créés échouent de manière sélective pour certaines personnes. En fin de compte, de nombreux services d'innovation éducative modernes semblent reproduire les inégalités conformément aux schémas historiques de discrimination raciale et ethnique attributive.

On pressent qu'un sentiment implicite d'impuissance puisse se développer lorsqu'on observe, dans le champ de la pratique, un flux apparemment incessant d'interventions et d'innovations n'accroissant pas l'équité. En dépit de tous ces investissements dans les innovations, les différences attributives prédisent encore trop souvent la réussite et les résultats. L'enseignement de la lecture en est un exemple parlant. Après des investissements excessivement importants visant à créer de nouvelles méthodes d'enseignement de la lecture, d'importantes lacunes subsistent dans notre capacité à enseigner la lecture aux élèves issus de groupes culturellement minoritaires par rapport aux groupes culturellement dominants. Pourquoi de nombreuses innovations ne tiennent-elles pas leurs promesses et pourquoi certaines innovations contribuent-elles très probablement à reproduire les inégalités ? Les problèmes sont-ils trop importants et les interventions, quoique bien conçues, sontelles trop faibles pour avoir un impact durable sur les disparités et pour combler les écarts de réussite et de résultats?

\section{Comment en sommes-nous arrivés là et que pouvons-nous faire?}

Est-ce que ça marche ou pas ? Voici, fondamentalement, le dispositif empirique du secteur pour évaluer l'efficacité et l'utilité des réformes. Mais le résultat de la mise en ouvre d'une innovation complexe dans un contexte complexe est plus nuancé que cela. En procédant de la sorte, le secteur laisse la porte ouverte à des innovations semblant à première vue efficaces, mais ayant une très grande variabilité s'agissant des groupes auxquels elles sont utiles et de ceux auprès desquels elles échouent. La stratégie du « est-ce que ça marche? » peut occulter les défaillances ou les performances peu élevées dans certains contextes scolaires particulièrement difficiles.

Considérons par exemple Sesame Street ${ }^{1}$, l'une des premières innovations destinées à favoriser la littératie chez les enfants défavorisés. Généralement saluée comme une réussite, cette innovation n'a en fait pas atteint ceux à qui elle était initialement destinée. Si les familles des classes moyennes et supérieures l'ont largement utilisée, elle a été sous-utilisée par les enfants et les familles défavorisés (Cook, Appleton, Conner, Shaffer, Tamkin et Weber, 1975 ; Cook et Conner, 1976).

1. Sesame Street est une série télévisée éducative américaine pour enfants diffusée à partir de 1969 sur le réseau PBS. Elle a été adaptée et/ou doublée dans plusieurs pays, dont la France. (NdT) 
Une autre innovation bien conçue - la Khan Academy ${ }^{2}$ - recèle la promesse d'un pouvoir révolutionnaire pour certains des élèves les plus défavorisés au monde, en rendant largement accessible des ressources pédagogiques de qualité. Des entreprises de télévision par câble et de services internet tels que Comcast se sont associées à la Khan Academy, dans le cadre du programme Comcast Internet Essentials, afin d'offrir un service internet à bas prix aux familles à faibles revenus, tout en permettant aux élèves d'utiliser les ressources de la Khan Academy ainsi que d'autres applications, dans l'objectif de lutter contre la fracture numérique. Une étude récente sur l'usage de la Khan Academy (Murphy, Gallagher, Krumm, Misley et Hafter, 2014) fait état d'un accueil généralement positif de la part des enseignants et des élèves, mais constate également des variations importantes de son utilisation d'un site à l'autre. Cette étude indique en outre que, contrairement au public d'élèves auquel elle s'adresse, une majorité d'enseignants estimait que la Khan Academy pourrait être très utile à leurs élèves les plus avancés. Seuls $25 \%$ des enseignants interrogés ont estimé que les ressources de la Khan Academy pourraient s'avérer efficaces pour les élèves les plus en retard en mathématiques au sein de leur groupe d'âge. De façon peut-être plus fréquente que ce l'on aurait naïvement anticipé, les innovations conçues pour aider ceux qui en ont le plus besoin, comme la Khan Academy, font souvent l'objet d'une sous-utilisation voire, potentiellement, d'une utilisation différentielle préjudiciable.

Nous (Bryk, Gomez, Grunow et LeMahieu, 2015) avons proposé un paradigme alternatif en matière d'efforts de recherche et de développement, visant à déceler de manière systématique pour qui, et dans quelles conditions, les interventions sont intéressantes. Pour cela, nous devons prêter une attention particulière aux micro-comportements au sein des processus et des routines. En somme, nous devons nous préoccuper des objectifs généraux et des éléments du système devant être articulés ensemble pour atteindre ces objectifs. Des objectifs ambitieux ne seront atteignables que lorsque des ensembles complexes de processus fonctionneront ensemble de manière harmonieuse. L'échec est susceptible de survenir à chaque point de connexion ou de transition au sein de cet ensemble de processus. Pour aborder l'équité par des innovations et des réformes, nous devons toujours garder à l'esprit la variabilité des résultats. Cela signifie que les concepteurs d'innovations éducatives doivent garder à l'esprit - ce qui semble évident - que les interventions connaissent rarement un succès ou un échec total. La variabilité des résultats est un sous-produit naturel de la rencontre entre une intervention et des systèmes organisationnels complexes (Fixen, Blasé et Fixen, 2017). En tant que telle, la clé pour produire des innovations plus réussies consiste à mettre en évidence la manière dont les processus de mise en œuvre engendrent l'échec pour certains et non pour d'autres. Pour déceler ces tendances, notre secteur doit s'engager différemment dans la mise en œuvre, afin que les interventions soient adaptées à l'échelle, pour fonctionner du premier coup et à chaque fois.

2. La Khan Academy est une association à but non lucratif fondée en 2008 visant à « fournir un enseignement gratuit et de qualité pour tout le monde, partout ». Le site publie en ligne un ensemble gratuit de mini-leçons, via des tutoriels vidéo YouTube. (NdT) 


\section{Comprendre les contextes et observer les processus}

Chaque fois qu'une innovation est introduite dans une école ou dans une organisation quelle qu'elle soit, débute ce que Schön (1992) appelle une « conversation » de conception avec le cadre, y compris ses usagers, leur histoire, leurs croyances antérieures, leurs préjugés et les outils actuels qu'ils utilisent pour faire leur travail. Nous nous inspirons de la littérature sur les interactions homme-machine ${ }^{3}$ pour définir ces interactions en tant que conversations de conception, car les usagers, lorsqu'ils sont confrontés à une innovation, sont chargés d'essayer de l'adapter à leur contexte. En substance, lorsqu'on demande aux gens de mettre en œuvre une nouvelle réforme, ils s'engagent dans une forme de recherche de sens ${ }^{4}$, afin de déterminer de quelle façon la nouvelle réforme sera spécifiquement intégrée à leurs routines de travail existantes. Il est important de garder à l'esprit que la recherche de sens est ici un acte d'interprétation qui engage les histoires, les aspirations et les croyances des individus. Il devient dès lors primordial d'expliciter clairement ce que l'on est censé faire (i.e. l'intervention elle-même), pourquoi (i.e. les principes de conception sous-jacents) et comment ceux-ci se manifestent dans les artefacts, les processus de travail et les normes sociales. C'est la raison pour laquelle chaque aspect de l'innovation se présente spécifiquement (plutôt que génériquement) tel qu'il est. Une telle spécificité détaillée informe le processus conversationnel qui se déroule dans un lieu par rapport à un autre. Et cela façonne en retour, au moins en partie, la variation que nous constatons dans la pratique et les résultats.

Le produit de la recherche de sens est l'ensemble des innombrables décisions que chaque praticien prend finalement pour donner vie à une intervention in situ. Même une intervention comportant un ensemble de processus relativement restreint nécessite une coordination importante pour être exécutée de manière fiable. Prenons, par exemple, le cas fictif d'une nouvelle intervention en mathématiques, nécessitant de porter attention à trois éléments de processus distincts, tels que la sélection des problèmes que les enseignants doivent mettre en œuvre pour utiliser l'intervention de façon concluante. Supposons en outre que les enseignants puissent effectivement mettre en œuvre chacun de ces éléments dans environ $90 \%$ des cas. Comme la fiabilité de l'ensemble du processus est le produit croisé de chaque processus, l'ensemble du processus sera mis en œuvre avec une efficacité d'environ $73 \%$. Ce niveau serait bien plus bas s'il existait, par exemple, des interdépendances entre les enseignants. On voit donc aisément comment un processus de mise en œuvre peut mal tourner, même pour une intervention relativement simple, produisant dès lors des résultats pouvant ne pas être efficaces de manière fiable pour tous les élèves.

Lorsque les interventions sont exécutées de manière peu fiable, l'impact peut ne pas être uniforme dans l'ensemble des contextes scolaires. La prise en compte de la fiabilité est particulièrement importante si l'on entend corriger les inégalités persistantes des résultats scolaires. Quelle que soit la qualité de l'intervention

3. Human Computer Interaction $(\mathrm{HCl})$, dans le texte original. (NdT)

4. Sense-making dans le texte original. (NdT) 
proposée, Payne $(1984 ; 2007)$ suggère par exemple qu'elle échouera souvent dans les contextes urbains, où bon nombre des élèves les plus défavorisés de notre pays ont tendance à être regroupés. De même, Shouse et Mussoline (1999) suggèrent que les interventions pédagogiques complexes ont tendance à ne pas être efficaces là où les infrastructures sociales et professionnelles sont faibles, comme c'est le cas dans les écoles aux ressources insuffisantes qui accueillent des enfants pauvres et issus de minorités. L'utilisation efficace d'une intervention exige un capital professionnel et social (Fullan, Rincón-Gallardo et Hargreaves, 2015 ; Hargreaves et Fullan, 2015) qui tend à être très rare dans les endroits caractérisés par des niveaux élevés de dysfonctionnement organisationnel (Chin, Lin et Chang, 2009). Dans ce contexte, la question clé est de savoir comment les organisations scolaires peuvent remédier aux dysfonctionnements de mise en ouvre et commencer à obtenir des résultats d'interventions plus fiables.

\section{LES ORGANISATIONS À HAUTE FIABILITÉ (HIGH RELIABLE ORGANIZATIONS)}

Lorsqu'un établissement persiste, par exemple, à appliquer un curriculum, alors que les enfants qui le suivent ne lisent pas assez bien pour l'utiliser efficacement, il y a là une forme de non-sens. Les comportements irréfléchis naissent lorsque règles et routines régissent les comportements sans qu'une attention suffisante soit portée à la situation présente. L'irréflexion est évidente dans les cas d'échec de mise en œuvre organisationnelle rendant inefficaces des interventions prometteuses pour les enfants pauvres. Pour lutter contre le non-sens, les organisations scolaires et les autres organisations doivent devenir conscientes.

Weick et al. (Weick, Sutcliffe et Ostifeld, 2005) se réfèrent à ce qu'ils appellent les organisations à haute fiabilité (High Reliable Organizations : HRO) comme exemple emblématique de lieux qui combattent efficacement l'irréflexion organisationnelle. L'expression « organisation à haute fiabilité » (OHF) est souvent employée dans des contextes institutionnels comme les centrales électriques, l'aviation et les sous-marins, où les défaillances graves doivent être évitées à tout prix. Le langage de la fiabilité n'est généralement pas utilisé en éducation. En matière d'éducation, on emploie plutôt le terme de fidélité. ${ }^{5}$

La fiabilité signifie ici que la performance d'une intervention est cohérente à travers une grande variété de contextes et qu'elle répond de façon satisfaisante aux besoins d'un grand nombre de personnes. En éducation, le langage de la fidélité va, dans la pratique éducative, jusqu'à la standardisation des intrants. Par exemple, au fil des ans, de nombreux districts scolaires ont entrepris de rédiger les cours pour faire en sorte que chaque cours soit dispensé exactement de la même manière par chaque enseignant. En substance, l'argument en faveur de la rédaction des cours est qu'en standardisant les intrants, des résultats de qualité surviendront de façon plus fiable.

5. En éducation, le concept de fidélité est issu de la psychométrie. Il implique l'homogénéité des questions d'un test, censées mesurer une même compétence; il implique donc aussi la standardisation des conditions de la mesure. $(N d I R)$ 
Dans d'autres secteurs, en revanche, les organisations à haute fiabilité se soucient à la fois de la cohérence des performances (résultats) et de la cohérence de la mise en ouvre (intrants). Freidman (2015) qualifie cette approche de responsabilité non seulement axée sur les résultats, mais aussi sur la mise à disposition d'intrants qui garantissent les résultats. Les organisations à haute fiabilité se concentrent sur l'apprentissage plutôt que sur la simple conformité. À mesure qu'elles apprennent, les OHF modifient de façon sélective les processus intrants en fonction des performances et du contexte. Par exemple, les compagnies aériennes peuvent disposer de processus standards sur la manière dont les pilotes doivent interagir lors de l'atterrissage d'un avion. Lorsque ces processus fonctionnent mal du fait de la configuration conceptuelle d'un nouvel appareil, les compagnies sont promptes à modifier les procédures d'atterrissage pour les adapter à la configuration de l'appareil (Hutchins, 1993), ce qui garantit une fiabilité continue quel que soit le contexte. De la même façon, lorsque les contextes locaux changent, par exemple lorsqu'un aéroport ne dispose que de courtes pistes d'atterrissage, les compagnies mettent automatiquement à jour leurs bases de données pour déterminer quels appareils de leur flotte peuvent atterrir en toute sécurité dans cet aéroport. Ce suivi des processus intrants, du contexte local et des performances est beaucoup moins courant dans les organisations éducatives.

Les idées de Weick et al. (2005) permettent de décrire ce que pourrait être un comportement de type OHF en éducation. Les OHF entretiennent une conscience organisationnelle - cinq dimensions spécifiques du comportement, que nous avons renommées et adaptées afin de les rendre plus accessibles et pertinentes pour le secteur de l'éducation. Ces cinq dimensions renommées sont les suivantes :

1) la prise en charge des échecs de performance ;

2) un engagement en faveur d'une conscience situationnelle partagée ;

3) la promotion de la capacité d'agir (agency) pour favoriser l'action ;

4) le sens de l'adaptation ;

5) la valorisation de la sagesse pratique et du raisonnement discipliné6.

La fiabilité en tant que principe d'organisation de l'action pourrait aider les réformateurs, les décideurs politiques et les usagers, comme les parents et les enseignants, à s'intéresser davantage à l'amélioration des innovations visant à atteindre l'égalité des résultats. En retour, ils devraient accepter moins volontiers les innovations produisant des résultats qui varient en fonction de conditions attributives telles que la pauvreté et la race. Dans les organisations à haute fiabilité, ces comportements encouragent les praticiens à s'exprimer et à agir, afin qu'ils demeurent attentifs à certaines caractéristiques clés du système tout en développant et en approfondissant la capacité d'action productive de tous.

6. Weick et al. ont établi un lien entre la conscience organisationnelle et les dimensions suivantes : (1) un intérêt pour l'échec, (2) une sensibilité publique aux opérations, (3) une structuration sous-spécifiée, (4) un engagement de résilience et (5) le rejet des solutions simplistes. Bien que le langage soit différent, chacune de ces dimensions entre en résonance avec des sujets d'actualité dans le champ éducatif. L'intérêt pour l'échec peut être aisément relié aux débats sur l'utilisation et la valeur des approches fondées sur les données probantes. La sensibilité publique aux opérations est liée, au moins en partie, à l'appel en faveur d'une pratique publique. La structuration sous-spécifiée entre en résonance avec l'intérêt du secteur éducatif pour l'élaboration de cultures participatives. L'engagement en faveur de la résilience peut être lié à l'idée que les personnes qui travaillent dans un système doivent avoir une perspective systémique afin de modifier le système. Enfin, il y a dans l'éducation un appel à résister à une conformité irréfléchie, c'est-à-dire que résister signifie le rejet des solutions simplistes issues de la sagesse conventionnelle, au profit d'une analyse davantage fondée sur des données probantes. 


\section{Apprendre à écouter différemment}

Afin de créer des systèmes qui fonctionnent bien dans les contextes locaux, les $\mathrm{OHF}$ doivent être à l'écoute des expériences vécues, qui peuvent contribuer à rendre les interventions opérationnelles en contexte. Être attentif à l'opérabilité contextuelle d'une intervention relève d'une sagesse éculée en matière de conception de toutes sortes d'artefacts. De façon moins commune, mais tout aussi essentielle, on peut considérer la centralité de l'usager comme une propriété des systèmes liée à l'équité. Par exemple, si nous pouvons observer ce qui s'est passé pour les utilisateurs d'une innovation qui a été perçue comme un échec, nous apprendrons probablement beaucoup plus en parlant avec les usagers qu'avec les personnes ayant fait l'expérience de l'échec. En l'absence de cette analyse au niveau du système, on court un risque élevé d'un biais d'attribution - qui aboutirait à considérer l'échec comme l'échec de certains individus, sans considérer les systèmes (processus et normes) au sein desquels ils fonctionnent.

La détection des problèmes est l'équivalent organisationnel de l'écoute systémique (Keisler et Sproull, 1982). Dans les cas d'échecs comme de succès, le défi consiste à déterminer comment parvenir à écouter largement. Un bon travail de détection de problèmes implique de voir la variation dans les échecs et de concevoir la fiabilité en réponse à celle-ci.

La capacité à détecter les échecs et à les relier aux caractéristiques du système s'illustre dans les pratiques des OHF. Les OHF s'intéressent aux processus en cours, en particulier ceux conduisant à des échecs. La pratique des OHF nous permet d'explorer de quelle façon l'hyper-vigilance pourrait conduire à des résultats plus équitables et plus fiables dans les systèmes éducatifs. L'Université d'État de Géorgie (Georgia State University : GSU) est un exemple d'organisation éducative s'intéressant aux données issues des performances. Afin d'identifier les obstacles les plus courants aux progrès et à la réussite des étudiants, la direction de l'université a créé un système de pairs mentors et de conseillers de faculté ayant recours à de simples rencontres avec les étudiants (pour leur rappeler par exemple d'assister aux cours) et à des discussions plus complexes, par exemple pour identifier et gérer les difficultés individuelles afin de comprendre les obstacles aux progrès des étudiants. En utilisant les informations tirées des conversations avec les étudiants et du comportement en matière d'assiduité des étudiants sur dix ans, ils ont créé un système de plus de 800 codes d'alerte différents visant à identifier les comportements à risque sur la base des activités quotidiennes des étudiants. En 2016, le système a fait plus de 51000 suggestions de rencontres pour intervenir en soutien aux progrès et à la réussite des étudiants. De manière impressionnante, les taux d'obtention de diplômes de la GSU ont augmenté de 36 points pour les Latinos et de $29 \%$ pour les Noirs ; en 2016, ces taux étaient de $58 \%$ pour les étudiants noirs et latinos.

[L']année dernière, les étudiants afro-américains, hispaniques, de première génération $^{7}$ et éligibles au programme de subventions fédéral Pell ${ }^{8}$ ont tous obtenu leur

7. En anglais, le terme « first-generation students » désigne les étudiants dont les parents n'ont pas fait d'études universitaires. (NdT)

8. Federal Pell Grant Program : programme de subventions que le gouvernement fédéral américain accorde aux étudiants qui en ont besoin pour financer leurs études universitaires. (NdT) 
diplôme de la GSU à des taux égaux ou supérieurs à l'ensemble du corps étudiant, ce qui fait de la GSU la seule université publique nationale à atteindre cet objectif. (Complete College Georgia) ${ }^{9}$

\section{Favoriser la capacité à agir là où elle est nécessaire}

Dans les OHF, le processus de désignation des décideurs importants est élargi et flexible, allant au-delà du leadership formel et faisant varier les personnes ayant accès à l'information sur la base de l'expertise et de l'expérience nécessaires sur le moment. Les OHF considèrent que la responsabilité de l'amélioration est collective. De cette façon, le leadership organisationnel propose un principe organisationnel important : le principe de subsidiarité, selon lequel les décisions devraient être prises par l'autorité compétente la plus locale et la moins centralisée, plutôt que par une structure de gestion plus traditionnelle, centralisée et descendante. En évitant les hiérarchies strictes, les OHF accélèrent le partage et la circulation de l'information au sein de l'ensemble de l'organisation. Ainsi, le système de conseil de la GSU diffuse simultanément des alertes aux conseillers de faculté, aux étudiants et aux pairs mentors. Chaque groupe comprend alors que le système tente de prévoir une rencontre afin d'aborder un sujet problématique.

\section{VigILANCE ET CONSCIENCE ORGANISATIONNELLES}

Adapter les interventions pour qu'elles soient utiles au plus grand nombre possible de personnes de manière fiable n'est pas une tâche aisée. Nous pensons que cela exige a minima d'adopter une nouvelle perspective sur la fiabilité en tant que telle. Jusqu'à présent, la fiabilité n’a pas été un principe directeur du développement des interventions éducatives. Sans un engagement en termes de fiabilité, il est possible de se contenter d'interventions qui ne fonctionnent bien que pour certains, ou de se reposer confortablement sur l'idée que l'inégalité des résultats est pratiquement impossible à éliminer, du fait des inégalités structurelles. Sur la base de notre lecture des OHF, nous estimons qu'un engagement en faveur de la fiabilité des performances peut changer les règles du jeu dans le champ éducatif. Plutôt que de se contenter de la fidélité de la mise en œuvre, les OHF se fixent un enjeu crédible et réalisable consistant à définir comme objectif l'atteinte de résultats de qualité pour tous. Ce faisant, les OHF peuvent pousser le champ éducatif à évoluer d'une approche axée sur les grands intrants à une approche axée sur les résultats consécutifs.

Évoluant dans des environnements où plane sans cesse le spectre d'une catastrophe, les OHF doivent être attentives aux échecs et adopter des postures organisationnelles leur permettant de détecter les défaillances et de réagir avec souplesse. $\mathrm{Si}$, dans l'ensemble, les échecs progressent plus lentement dans le champ éducatif, ils ne sont pas moins catastrophiques. Les organisations éducatives pourraient

9. http://www.completegeorgia.org/georgia-state-university-campus-plan-update-2016 
également adopter la fiabilité comme principe directeur de leur action organisationnelle. Le secteur éducatif pourrait chercher à développer une vigilance et une conscience organisationnelles similaires à celles développées dans les OHF. Dans le secteur éducatif, une telle vigilance nous encourage à analyser minutieusement les échecs prévisibles et à les appréhender sous un autre angle. Les organisations éducatives dont nous avons ici souligné les réalisations en sont venues à considérer les défaillances graves comme devant, par nature, être éliminées de la pratique. Dans cet article, nous suggérons qu'afin de faire évoluer nos discours sur l'échec et ses liens avec la classe sociale, la pauvreté, l'ethnicité et la race, nous devrions adopter un tel état d'esprit. En bref, lorsqu'une intervention échoue pour les enfants de familles à faibles revenus à des taux qui dépassent de loin le taux d'échec de leurs camarades issus de milieux plus aisés, nous devrons proclamer que ce n'est là ni l'objectif ni la norme que nous désirons. Dans des OHF éducatives, en effet, une telle incidence mobiliserait les ressources organisationnelles afin de comprendre et de faire cesser les échecs prévisibles.

\section{RÉFÉRENCES BIBLIOGRAPHIQUES}

BRYK A., GOMEZ L., GRUNOW A. et LEMAHIEU P. (2015). Learning to improve: How America's Schools Can Get Better at Getting Better. Cambridge : Harvard Education Press.

CHEN Y-S., LIN M-J. et CHANG C-H. (2009). "The positive effects of relationship learning and absorbtive capacity on innovative performance and competitive advantage in industrial markets ». Industrial Marketing Management, $\mathrm{n}^{\circ} 38$ (2), p. 152-158.

COOK T. D., APPLETON H., CONNE R., SHAFFER A., TAMKIN G. et WEBER S. J. (1975). Sesame Street Revisited. Russell Sage Foundation.

COOK T. et CONNER R. (1976). « The Educational Impact ». Journal of Communication, $n^{\circ} 26$ (2), p. 155-164.

FIXSEN A., BLASE K. et FIXSEN D. (2017). Four best practices for scaling up effective innovations. Chapel Hill : Frank Porter Graham Child Development Institute, The University of North Carolina at Chapel Hill.

FULLAN M., RINCÓN-GALLARDO S. et HARGREAVES A. (2015). «Professional capital as accountability ». Education Policy Analysis Archives, $\mathrm{n}^{\circ} 23$ (15).

HARGREAVES A. et FULLAN M. (2015). Professional capital: Transforming teaching in every school. Teachers College Press.

HUTCHINS E. (1993). "Learning to navigate ». Dans CHAIKLIN S. et LAVE J., Understanding Practice: Perspectives on Activity and Context, Cambridge : Cambridge University Press, p. 35-63.

KIESLER S. et SPROULL L. (1982). « Managerial Response to Changing Environments: Perspectives on Problem Sensing from Social Cognition ». Administrative Science Quarterly, nº 27 (4), p. 548-570.

MURPHY R., GALLAGHER L., KRUMM A.E., MISLEVY J. et HAFTER A. (2014). "Research on the Use of Khan Academy in Schools: Research Brief ». Menlo Park : SRI International. En ligne. [https://bit.ly/2xOpdse]

PAYNE C.M. (2007). I've got the light of freedom: The organizing tradition and the Mississippi freedom struggle. University of California Press. 
PAYNE C.M. (1984). Getting What We Ask For: The Ambiguity of Success and Failure In Urban Education. Contributions to the Study of Education (Book 12), Praeger.

SHOUSE R.C. et MUSSOLINE L.J. (1999). «High risk, low return: The achievement effects of restructuring in disadvantaged schools ». Social Psychology of Education, $\mathrm{n}^{\circ}$ 3(4), p. 245-259.

WEICK K., SUTCLIFFE K. et OBSTFELD D. (2005). " Organizing and the process of sense-making ». Organization Science, $n^{\circ} 16$ (4), p. 409-421. 